\title{
Desfecho clínico de pacientes colonizados e infectados por bactérias multirresistentes em hospital privado de Fortaleza/Ceará, em 2021
}

\author{
Clinical outcome of patients colonized and infected by multidrug-resistant bacteria in a private \\ hospital in Fortaleza/Ceará, in 2021 \\ Evolución clínica de pacientes colonizados e infectados por bacterias multirresistentes en un \\ hospital privado de Fortaleza/Ceará, en 2021
}

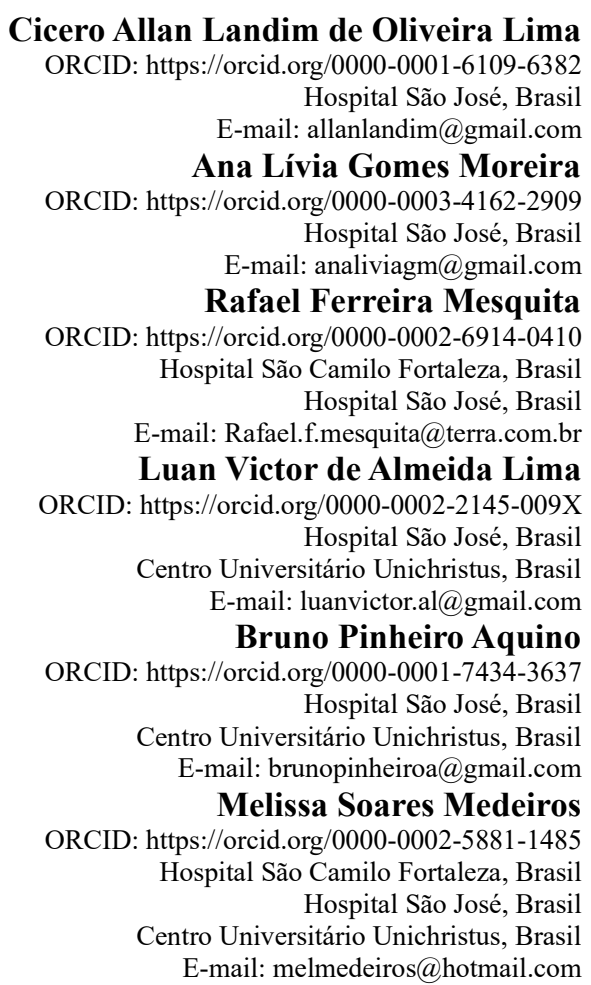

\section{Resumo}

A pandemia pela COVID-19, desde 2019, vem contribuindo para agravar situações que aumentam o risco de desenvolvimento de colonização ou infecção por bactérias multirresistentes, como o uso indiscriminado de antimicrobianos. O objetivo deste estudo foi investigar a relação entre resistência bacteriana e desfecho clínico negativo. Foi realizado estudo do tipo transversal e retrospectivo, onde foram coletados dados dos prontuários de pacientes internados em diferentes unidades hospitalares (UTI, enfermaria, isolamento e neonatologia), de janeiro a dezembro de 2021. Os critérios de inclusão foram os pacientes notificados com colonização ou infecção por bactérias multirresistentes durante o internamento e com dados completos. Foram 128 amostras positivas, com média de idade 68,2 anos. Houve diferença estatisticamente significante em mortalidade ao comparar pacientes com menos de 50 anos e os grupos de faixa etária entre 70-80 anos $(\mathrm{p}=0,006)$ e acima de 80 anos $(\mathrm{p}=0,03)$. Dentre os três principais patógenos isolados e a elevada taxa de mortalidade dos mesmos, não houve diferença estatisticamente significativa em relação a taxa de mortalidade global para Acinetobacter baumannii $(\mathrm{p}=0,54)$, Klebsiella pneumoniae $(\mathrm{p}=1)$ e Pseudomonas aeruginosa $(\mathrm{p}=0,82)$. Detectou-se resultado estatisticamente significativo para mortalidade e utilização de quinolonas $(p=0,047)$ e uma tendência a significância para polimixina $(p=0,09)$. Concluiu-se que houve elevada mortalidade em pacientes colonizados ou infectados por bactérias multirresistentes em âmbito hospitalar, principalmente acima de 70 anos de idade. O uso de quinolonas, aminoglicosídeos e polimixina parece estar relacionado a maior mortalidade.

Palavras-chave: Antimicrobianos; Bactéria; Mortalidade; Colonização; Infecção. 


\begin{abstract}
The COVID-19 pandemic, since 2019, has contributed to aggravating situations that increase the risk of colonization or infection by multidrug-resistant bacteria, such as the indiscriminate use of antimicrobials. The aim of this study was to investigate the relationship between bacterial resistance and negative clinical outcome. A cross-sectional and retrospective study was carried out, where data were collected from the medical records of patients hospitalized in different hospital units (ICU, ward, isolation and neonatology units), from January to December 2021. Inclusion criteria were patients reported with colonization or infection by multidrug-resistant bacteria during hospitalization and with complete data. There were 128 positive samples, with a mean age of 68.2 years. There was a statistically significant difference in mortality when comparing patients younger than 50 years and the age groups between 70-80 years $(p=0.006)$ and above 80 years $(p=0.03)$. Among the three main pathogens isolated and their high mortality rate, there was no statistically significant difference in terms of the overall mortality rate for Acinetobacter baumannii $(\mathrm{p}=0.54)$, Klebsiella pneumoniae $(\mathrm{p}=1)$ and Pseudomonas aeruginosa $(\mathrm{p}=1) .=0.82)$. A statistically significant result was detected for mortality and use of quinolones $(\mathrm{p}=0.047)$ and a trend towards significance for polymyxin $(\mathrm{p}=0.09)$. It was concluded that there was high mortality in patients colonized or infected by multidrug-resistant bacteria in a hospital setting, mainly over 70 years old. The use of quinolones, aminoglycosides and polymyxin seems to be related to higher mortality.
\end{abstract}

Keywords: Antimicrobials; Bacterium; Mortality; Colonization; Infection.

\title{
Resumen
}

La pandemia de COVID-19, desde 2019, ha contribuido a agravar situaciones que aumentan el riesgo de colonización o infección por bacterias multirresistentes, como el uso indiscriminado de antimicrobianos. El objetivo de este estudio fue investigar la relación entre la resistencia bacteriana y el resultado clínico negativo. Se realizó un estudio transversal y retrospectivo, donde se recogieron datos de las historias clínicas de pacientes hospitalizados en diferentes unidades hospitalarias (UCI, sala, aislamiento y neonatología), de enero a diciembre de 2021. Los criterios de inclusión fueron pacientes reportados con colonización o infección por bacterias multirresistentes durante la hospitalización y con datos completos. Hubo 128 muestras positivas, con una edad media de 68,2 años. Hubo diferencia estadísticamente significativa en la mortalidad al comparar pacientes menores de 50 años y los grupos de edad entre 70-80 años ( $\mathrm{p}=0,006)$ y mayores de 80 años $(\mathrm{p}=0,03)$. Entre los tres principales patógenos aislados y su alta tasa de mortalidad, no hubo diferencia estadísticamente significativa en cuanto a la tasa de mortalidad global para Acinetobacter baumannii ( $\mathrm{p}=$ 0,54), Klebsiella pneumoniae $(\mathrm{p}=1)$ y Pseudomonas aeruginosa $(\mathrm{p}=1) .=0,82)$. Se detectó un resultado estadísticamente significativo para mortalidad y uso de quinolonas $(\mathrm{p}=0,047)$ y tendencia a la significación para polimixina $(p=0,09)$. Se concluyó que existe una alta mortalidad en pacientes colonizados o infectados por bacterias multirresistentes en un medio hospitalario, principalmente mayores de 70 años. El uso de quinolonas, aminoglucósidos y polimixina parece estar relacionado con una mayor mortalidad.

Palabras clave: Antimicrobianos; Bacteria; Mortalidad; Colonización; Infección.

\section{Introdução}

A resistência antimicrobiana é considerada um problema de saúde global, que compromete a efetividade dos antibióticos, inviabilizando o tratamento de infecções comuns, aumentando o tempo de internamento hospitalar e por muitas vezes levando os pacientes a desfechos negativos. A resistência ocorre quando microrganismos sofrem mutação genética ao serem expostos a drogas antimicrobianas, sendo referidos como multirresistentes ao considerarmos pelo menos três classes ineficientes contra estes patógenos (de Queiroz et al., 2012).

Atualmente a resistência antimicrobiana foi eleita pela Organização Mundial de Saúde como uma das dez maiores ameaças a saúde pública global. Estimando-se, que até 2050, o problema causará cerca de 10 milhões de mortes por ano, além de um grande prejuízo financeiro de cerca de 100 trilhões de dólares. Até 2030, a resistência antimicrobiana poderá forçar até 24 milhões de pessoas à pobreza extrema (ANVISA, 2019). Atualmente, pelo menos 700.000 pessoas morrem a cada ano devido a doenças causadas por patógenos resistentes aos tratamentos disponíveis (WHO, 2019). Um importante fator que viabiliza condições necessárias ao aumento da resistência antimicrobiana é o uso inadequado dos antimicrobianos na rotina hospitalar, principalmente nas unidades de terapia intensiva, associado a dificuldades na prática das rotinas de controle de infecção hospitalar.

Com a chegada da pandemia pela COVID-19 em 2019, foram incrementadas mais condições para disseminação desses patógenos. O uso indiscriminado dos antimicrobianos devido a não possibilidade de diferenciar infecção pelo Sars-Cov-2 de 
uma provável pneumonia comunitária em um primeiro momento, fez com que antimicrobianos de largo espectro fossem utilizados por períodos prolongados e sem critérios objetivos, muitas vezes de forma empírica. Há relatos de divergência da prevalência de infecção secundaria relacionada a Covid-19 na literatura, mas na faixa de 4,9\%. Portanto, há uma provável prescrição excessiva de antimicrobianos nessa infecção viral (Langford et al, 2021; ANVISA, 2021).

É importante salientarmos o maior risco que o paciente em cuidados críticos está submetido, seja pelo uso de dispositivos invasivos ou pelo maior risco de colonização. No caso dos pacientes infectados por Sars-Cov-2, é constante o número de pacientes com internamento prolongado e com maior prevalência de procedimentos invasivos, como a intubação orotraqueal, passagem de cateter venoso central e sonda vesical de demora.

O acrônimo "ESKAPE", refere-se às bactérias mais comumente associadas com a resistência a múltiplas drogas, incluindo Enterococcus faecium, Staphylococcus aureus, Klebsiella pneumoniae, Acinetobacter baumannii, Pseudomonas aeruginosa e Enterobacteriaceae. Estes microrganismos são considerados os principais agentes envolvidos em infecções hospitalares e efetivamente escapam dos efeitos das drogas antimicrobianas (Bassetti et al., 2013).

São definidas como multirresistentes as cepas de A. baumannii que produzem carbapenases e apresentam resistência contra pelo menos três classes de antimicrobianos. As cepas panresistentes conseguem se opor mesmo frente a antibacterianos de amplo espectro, como polimixina e tigeciclina (Queenan, et al., 2014). A infecção por este microrganismo em pacientes internados é um indicador de risco para doenças graves, sendo a alta mortalidade relacionada ao tratamento realizado nas Unidades de Terapia Intensiva (UTIs), quando o paciente em geral já apresenta fragilidade imunológica e necessidade de procedimentos invasivos para suporte de vida (Nascimento et al, 2021).

O desenvolvimento de novos antimicrobianos é complexo, pois além da dificuldade na descoberta de inovadores mecanismos de ação, exige anos de pesquisa e é extremamente dispendioso para as indústrias farmacêuticas. Esses fatores proporcionam um acesso restrito à população, pois quando novos fármacos são lançados chegam ao mercado com altos custos, causando aplicação não igualitária na sociedade. Logo, esses novos fármacos não solucionam a problemática por completo (de Queiroz et al., 2012).

Desde o ano 2000, poucas classes de antimicrobianos foram introduzidas no mercado para uso humano, tais como oxazolidinonas, lipopeptídeos e glicilciclinas. Os fármacos desenvolvidos mais recentemente, pertencem a novas classes ou oriundas de modificações em classes antigas, para o tratamento clínico de infecções causadas por microrganismos multirresistentes, liberados pelo Food and Drug Administration (FDA) na última década, foram: linezolida (2000), ertapenem (2001), daptomicina (2003), telitromicina (2004), tigeciclina (2005), doripenem (2007), ceftarolina (2010), ceftolozana/tazobactam (2014), ceftazidima/avibactam (2015), dentre outros (de Queiroz et al., 2012). Dessa forma, várias agências e sociedades de profissionais têm chamado a atenção para a necessidade de novos antimicrobianos, especialmente para as bactérias Gram negativas. Desde 2004, a Infectious Diseases Society of America (IDSA) tem reivindicado investimentos na indústria farmacêutica para o desenvolvimento de novos antimicrobianos (Bassetti et al., 2013). Devido a tal problemática e escassez de novos antimicrobianos, atualmente há a necessidade de utilização de drogas antigas, como a polimixina, para o tratamento dessas infecções, porém com o provável incremento de sua toxicidade.

A Organização Mundial de Saúde destaca em um relatório a necessidade de esforços coordenados e intensivos para superar a resistência antimicrobiana. Uma grande barreira para a realização de muitos dos "Objetivos de Desenvolvimento Sustentável" da ONU é a cobertura universal de saúde, obtenção de alimentos seguros, sistemas agrícolas sustentáveis, água potável e saneamento (WHO, 2019). Importante ressaltar o desequilíbrio ambiental na saúde da população, vide a constatação de casos de doenças que foram geradas, direta ou indiretamente, por lançamentos inadequados de efluentes, sendo as unidades prestadoras de Serviços de Saúde contribuintes para a degradação ambiental (da Silva, 2020). As infecções bacterianas decorrentes da resistência antimicrobiana também prosperam em condições de instabilidade civil, pobreza, migração de massa e 
degradação ambiental, principalmente quando há exposição as doenças infecciosas por grande número populacional aliada à ineficiência dos serviços de saúde (Costa, 2016).

O objetivo deste estudo é investigar a relação entre resistência a antibióticos e desfecho clínico negativo definido como óbito, pois é difícil discriminar os fatores de confusão e determinantes dessa relação. Além disso, os pacientes com maior risco de morte também são provavelmente aqueles com maior risco de infecção por bactérias altamente resistentes a antimicrobianos (Lakbar et al, 2021). Deste modo, é necessário que haja definição do perfil de resistência dos patógenos sabidamente causadores de infecções, bem como determinar como a colonização dos mesmos pode inferir na morbimortalidade. Além de reconhecer o impacto e importância das medidas de prevenção pelo uso racional de antimicrobianos.

\section{Metodologia}

\subsection{Tipo de estudo}

Foi realizado um estudo do tipo transversal e retrospectivo, onde foram coletados dados dos prontuários de pacientes internados no Hospital São Camilo de Fortaleza, uma instituição privada, composta por serviço de emergência clínica e obstétrica, enfermaria, Unidades de Terapia Intensiva adulta e neonatal, no período de janeiro a dezembro de 2021.

Estudos observacionais são aqueles em que o pesquisador está documentando uma relação natural entre a exposição e o resultado que está estudando. $O$ pesquisador não faz nenhuma intervenção ativa em nenhum indivíduo, e a exposição já foi decidida naturalmente ou por algum outro fator. Os estudos analíticos tentam testar uma hipótese e estabelecer relações causais entre as variáveis. Estudos analíticos podem ser observacionais (se a exposição for determinada naturalmente). Em estudos retrospectivos, o desfecho de interesse já ocorreu (ou não ocorreu - por exemplo, em controles) em cada indivíduo, e os dados são coletados de registros ou solicitando aos participantes que recordem as exposições. Não há acompanhamento dos participantes ao longo do tempo (Ranganathan et al, 2018). Portanto, o presente estudo se enquadra nestas características.

Os dados coletados foram referentes a infecções bacterianas multirresistentes e os desfechos clínicos. A amostra foi composta por todos os prontuários de pacientes incluídos no estudo durante todo o período avaliado. Para a pesquisa, a análise dos prontuários teve como finalidade a coleta dos seguintes aspectos: idade, sexo, tratamento, etiologia da infecção, e desfecho clínico.

\subsection{Local e período de estudo}

A pesquisa foi realizada no Hospital São Camilo (HSC), localizado na Rua Costa Barros, 833 - Centro, Fortaleza - CE, 60160-280. No período entre janeiro a dezembro de 2021.

\subsection{População do estudo}

Pacientes do Hospital São Camilo que, durante o período acima, apresentaram patógenos multirresistentes durante coleta de amostras de culturas (hemocultura, urinocultura, culturas de secreções respiratórias ou outras).

\subsection{Coleta de Dados}

Foram coletados os dados clínicos, laboratoriais e prescrições de antimicrobianos dos pacientes, buscando avaliar o impacto na mortalidade de patógenos multirresistentes.

\subsection{Critérios de inclusão}

Os critérios de inclusão foram os pacientes notificados com colonização ou infecção por bactérias multirresistentes durante o internamento e notificados pelo Serviço de Controle de Infecção Hospitalar (SCIH). 


\subsection{Critérios de exclusão}

Foram excluídos do estudo pacientes que não tiveram infecção por bactérias multirresistentes.

\subsection{Amostra}

Formada pela população de pacientes atendidos no HSC e que preencham os critérios de inclusão supracitados.

\subsection{Análise de dados}

Os dados nominais foram expressos em forma de gráficos. E análise estatística através do Graphpad online, aplicando teste de Fisher com 2 desvios padrões e IC de 95\%, considerando significante quando $p<0,05$.

\subsection{Aspectos éticos}

Esta pesquisa seguiu a Resolução 466/12 do Conselho Nacional de Saúde, que regulamenta pesquisas envolvendo seres humanos. O estudo foi aprovado pelo Comitê de Ética do Centro Universitário Christus/Unichristus com CAAE: 91385218.3.0000.5049 e parecer número 2.764.840.

\section{Resultados}

Foram 128 amostras coletadas e positivas para bactérias multirresistentes durante a internação de pacientes no ano de 2021. Destes pacientes, 07 apresentaram pelo menos duas culturas por microrganismos diferentes $(\mathrm{N}=121)$. Um paciente não apresentava dados para cálculo de idade no prontuário, e os gráficos relacionados foram calculados com total de 127 pacientes. Foram 62 do sexo feminino e 66 do sexo masculino. Média de idade 68,2 anos variando de 0 a 103 anos, sendo 2 recém-nascidos. Sobre a idade dos pacientes, destacou-se que a maioria se situava na faixa etária de 70 a 79 anos, seguido de pacientes maiores de 80 anos de idade. $O$ menor tempo médio de permanência encontrado foi no grupo acima de 80 anos de idade, sendo que $80 \%$ dos pacientes tiveram internamento em unidade de terapia intensiva em algum momento durante a internação hospitalar. Além disso, $46 \%$ dos pacientes totais apresentaram internamento entre 07 e 30 dias, seguido de $33 \%$ dos pacientes que se encontraram internados entre 30 a 60 dias, com tempo máximo detectado de 171 dias (Tabela 1).

Tabela 1. Estratificação por idade dos pacientes com infecção/colonização por bactérias multirresistentes associados a tempo de permanência e desfecho negativo em 2021.

\begin{tabular}{|l|l|c|c|c|}
\hline Idade (anos) & N & $\begin{array}{c}\text { Média de tempo de } \\
\text { internação (Dias) }\end{array}$ & $\begin{array}{c}\text { Desfecho Negativo } \\
\text { (Óbito) } \\
\mathrm{N}\end{array}$ & $\begin{array}{c}\text { Desfecho Negativo } \\
\text { (Óbito) } \\
\%\end{array}$ \\
\hline $0 \mathrm{a}<50$ & 16 & 46,2 & 3 & 18,7 \\
\hline $50 \mathrm{a}<60$ & 22 & 36,4 & 6 & 27,3 \\
\hline $60 \mathrm{a}<70$ & 19 & 46,2 & 7 & 36,8 \\
\hline $70 \mathrm{a}<80$ & 36 & 45,7 & 22 & 61,1 \\
\hline$>80$ & 34 & 38 & 18 & 52,9 \\
\hline TOTAL & 127 & 42,2 & 56 & 44 \\
\hline
\end{tabular}

Fonte: Gráfico construído a partir de dados dos autores. Apresentação dos dias de internação hospitalar e desfecho negativo (óbito) de acordo com a faixa etária de distribuição dos pacientes. 
Houve diferença estatisticamente significante em mortalidade ao comparar pacientes com menos de 50 anos e os grupos de faixa etária entre 70-80 anos ( $\mathrm{p}=0,006)$, e acima de 80 anos ( $\mathrm{p}=0,03)$, (Figura 1).

Figura 1. Avaliação por idade (anos) da mortalidade em pacientes colonizados/infectados por bactérias multirresistentes em 2021.

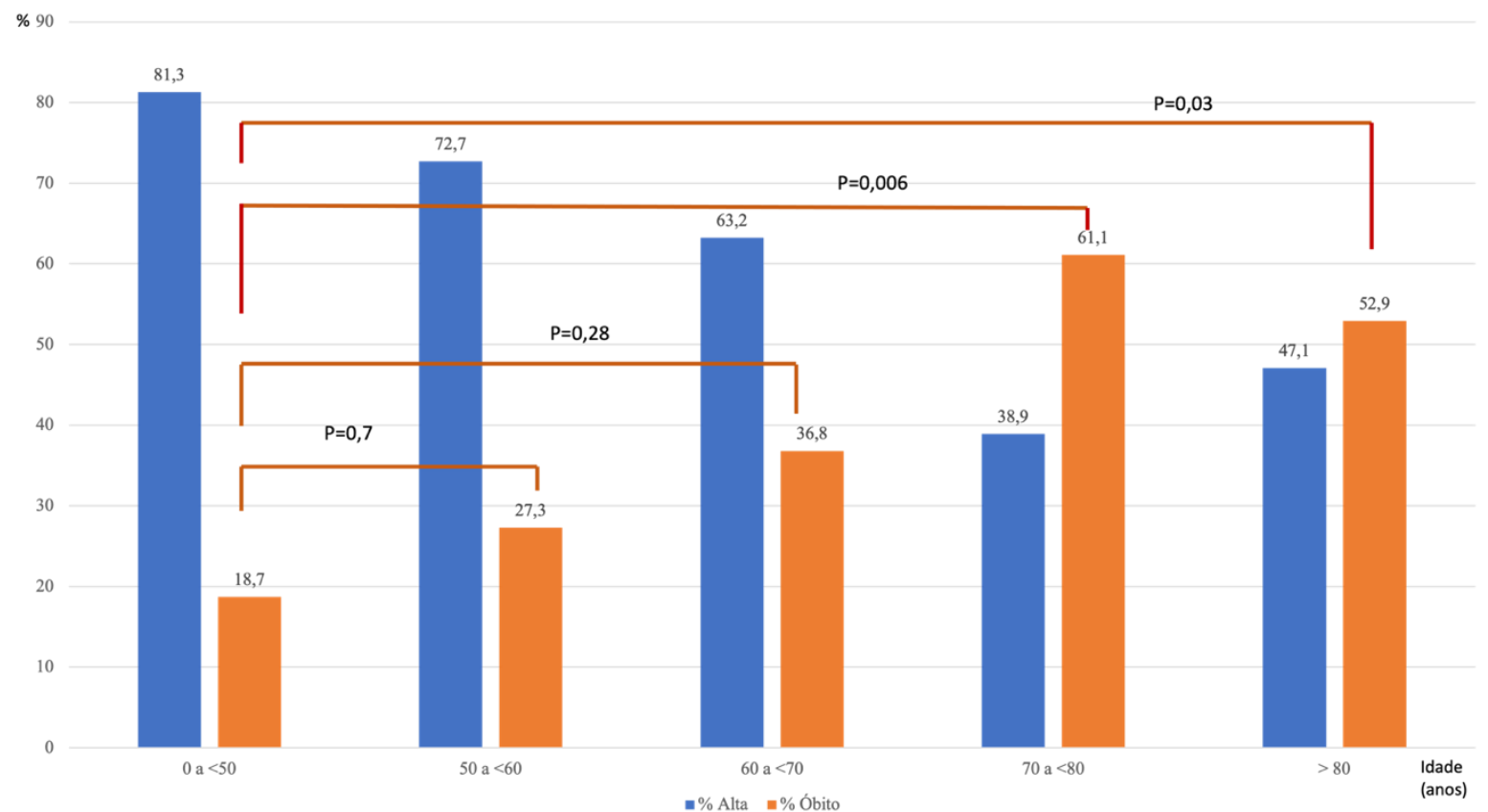

Fonte: Gráfico construído a partir de dados dos autores. Evidencia-se a estratificação por idade e impacto na mortalidade. Observa-se significância ao comparar pacientes abaixo de 50 anos e aqueles nas faixas etárias de 70 a 80 anos e acima de 80 anos de idade.

Do total de 128 culturas positivas, 95 foram determinadas através da pesquisa por swab retal para isolamento e 33 pacientes com microrganismos detectados de outros sítios (aspirado traqueal, urinocultura, hemoculturas e lavado broncoalveolar), sendo pacientes considerados como infectados ou colonizados (Figura 2).

Figura 2. Sítios de infecção ou colonização onde houve crescimento e detecção de patógenos multirresistentes em 2021.

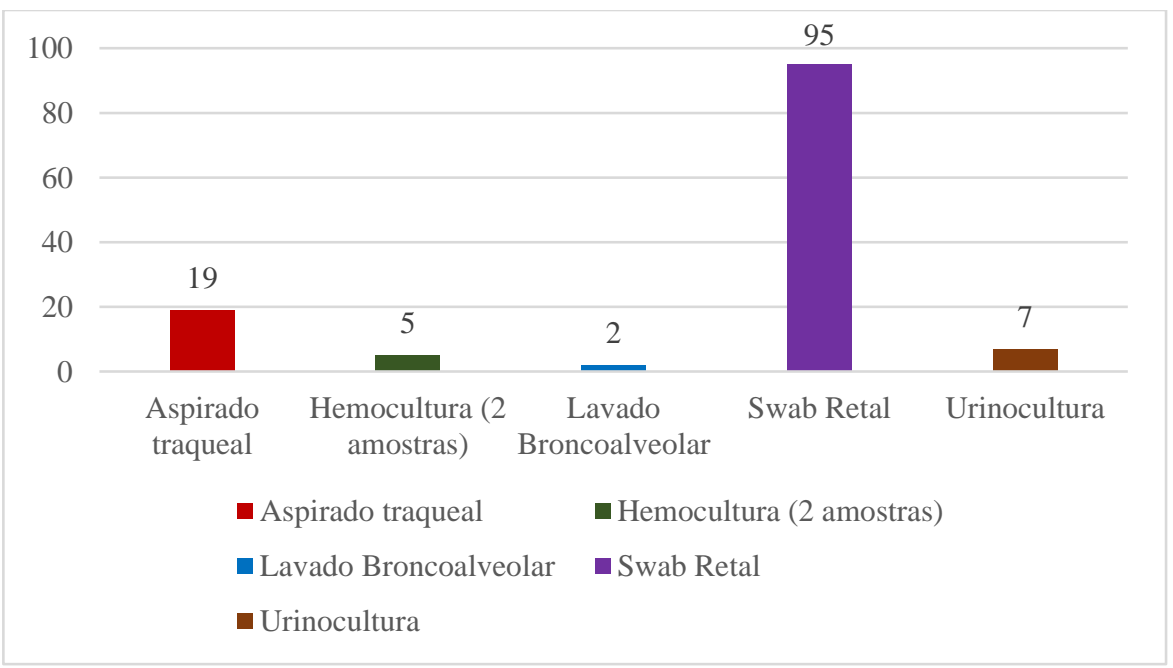

Fonte: Gráfico construído a partir de dados dos autores. Observa-se o maior crescimento em swab retal das amostras identificadas com patógenos multirresistentes, seguida de aspirado traqueal que somando-se as amostras de lavado broncoalveolar representam o percentual de infecções pulmonares na instituição. 
Foram identificadas amostras em 115 dos pacientes (89\%), sendo os demais (11\%) resultado obtido através de testes rápidos para enzimas KPC e VRE em culturas de vigilância. A Figura 3 representa o número de amostras obtidas ao mês durante o ano de 2021.

Figura 3. Isolamento de bactérias multirresistentes distribuídas durante o ano de 2021.

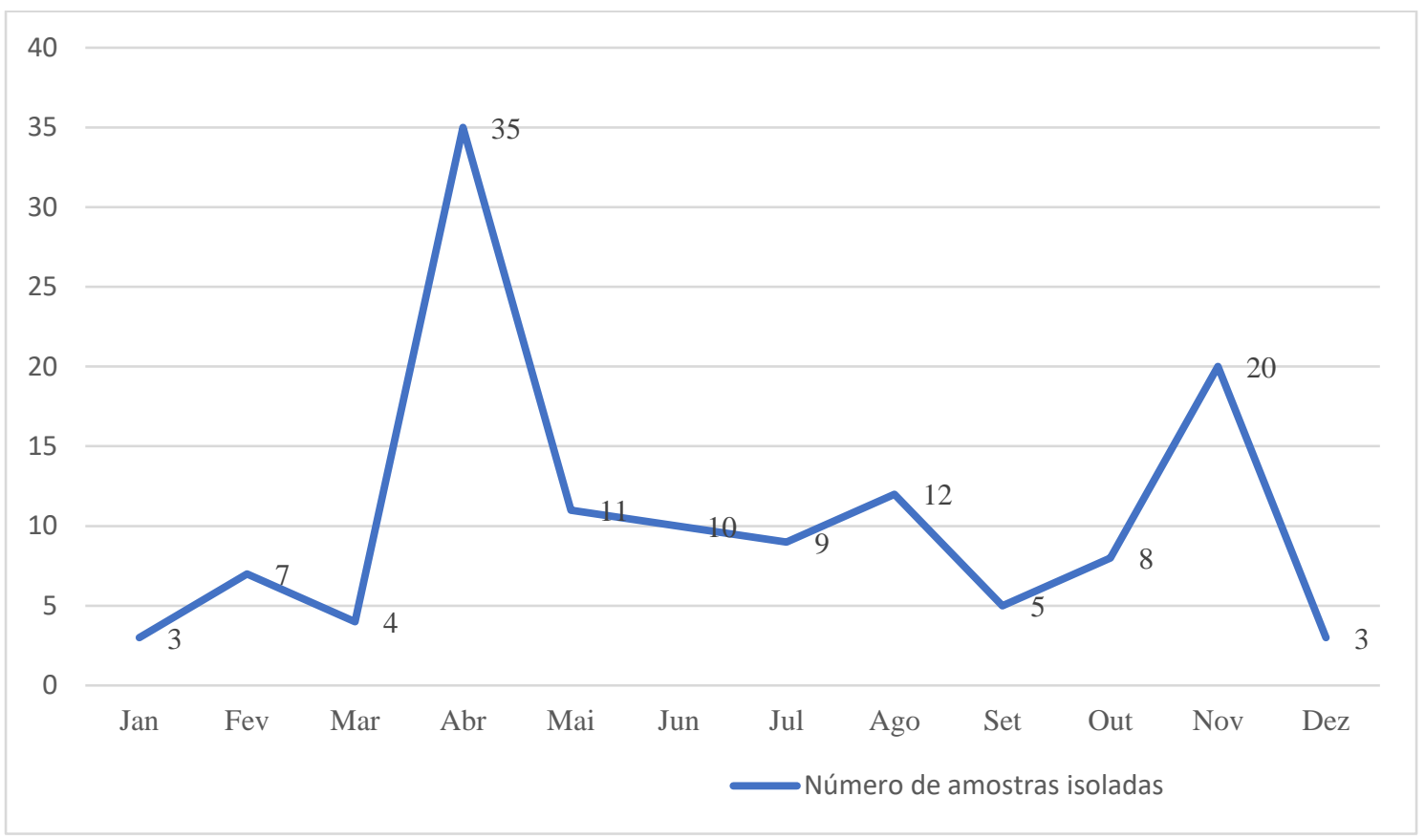

Fonte: Gráfico construído a partir de dados dos autores. A figura representa o número de amostras que positivaram de acordo com os meses de 2021 para bactérias multirresistentes. Evidencia-se aumento no período de março a maio, onde pacientes críticos crônicos permaneceram mais tempo em tratamento antimicrobiano de amplo espectro.

Foram detectados resultados positivos para K. pneumoniae $(\mathrm{N}=71)$, P. aeruginosa $(\mathrm{N}=24)$, A. baumannii $(\mathrm{N}=13)$, Serratia marcecens $(\mathrm{N}=3)$, E. cloacae $(\mathrm{N}=2)$, E. coli $(\mathrm{N}=2)$, E. Faecalis $(\mathrm{N}=1)$ e S. aureus $(\mathrm{N}=1)$, (Figura 4). Do número total de culturas positivas obtidas por swab retal ( $\mathrm{N}=95), 20 \%$ foram identificadas como Pseudomonas aeruginosa $(\mathrm{N}=19), 57,9 \%$ como $K$. pneumoniae $(\mathrm{N}=55)$ e 5,3\% como A. baumannii $(\mathrm{N}=5)$. Destes, a maior mortalidade foi atribuída a colonização ou infecção por K. pneumoniae. Foram definidos como KPC 111 amostras, multirresistência 15, e panresistência e VRE uma amostra cada. 
Figura 4. Identificação de bactérias multirresistentes isoladas de amostras coletadas durante o ano de 2021.

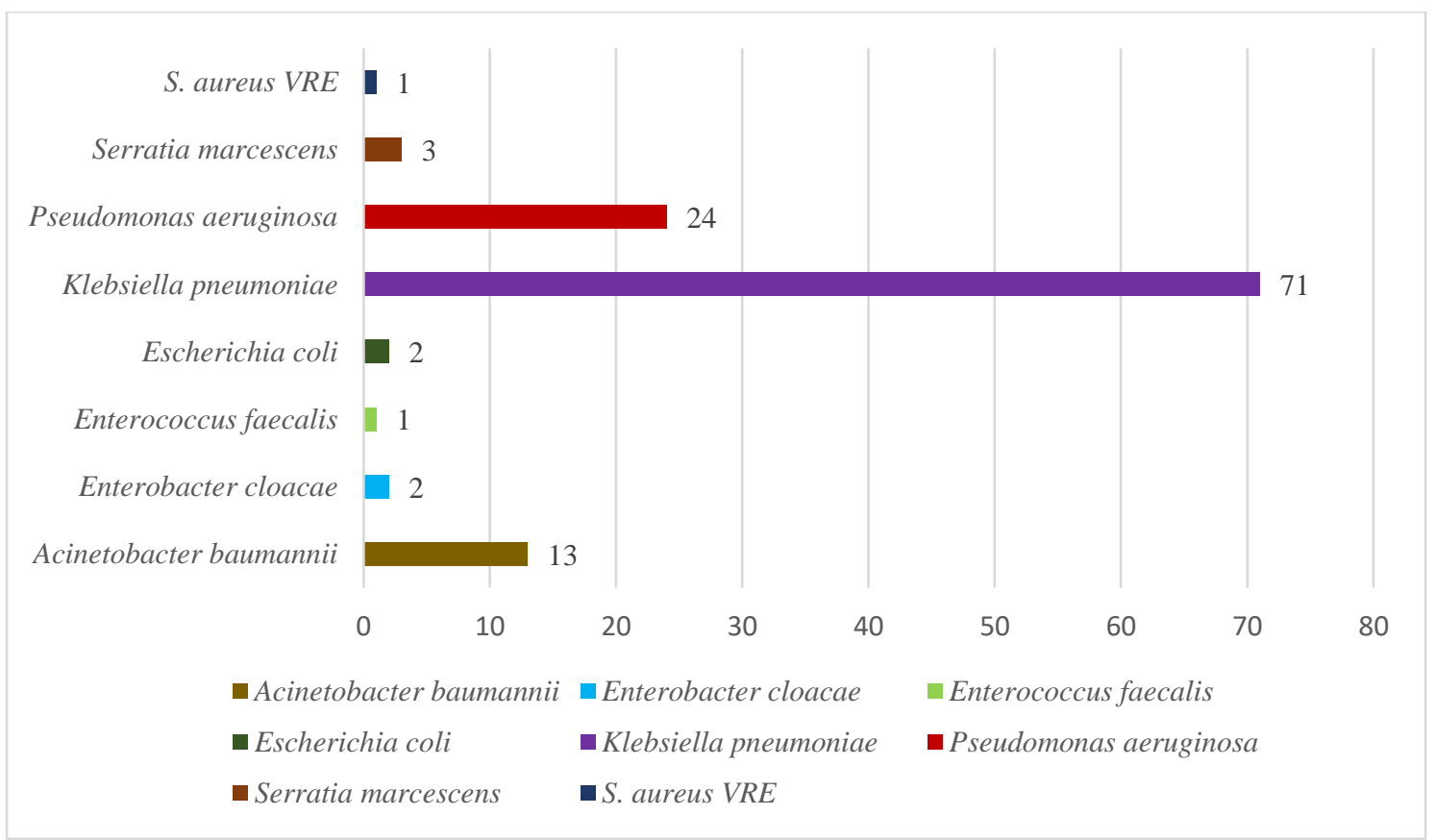

Fonte: Gráfico construído a partir de dados dos autores. O gráfico mostra o número absoluto de bactérias multirresistentes isoladas das amostras totais, observando-se a alta prevalência de $K$. pneumoniae, $P$. aeruginosa e A. baumannii.

Dentre os antimicrobianos utilizados durante a internação dos pacientes de controle pelo serviço de controle de infecção hospitalar (SCIH) detectados em 415 prescrições, a piperacilina-tazobactam foi o antibiótico mais utilizado, tendo sido prescrito em $18,8 \%$ destes pacientes $(\mathrm{N}=78)$. A seguir, temos o grupo dos glicopeptídeos como uma das classes de antimicrobianos mais prescrita para os pacientes elencados, tendo sido utilizados em 18,3\% dos pacientes (38 utilizaram teicoplanina, 07 utilizaram vancomicina e 16 alternaram uso entre ambos). Ainda com relação a medicamentos com potencial cobertura para Gram positivos, 8,6\% pacientes fizeram uso de de linezolida $(\mathrm{N}=36)$, antimicrobiano pertencente a classe das oxazolidinonas e com boa ação contra VRE. O uso de daptomicina, um lipopeptídeo, ficou restrito a 2,6\% dos pacientes (N=11), (Figura 5).

Segue-se em relação a ordem de prescrição, o uso de carbapenêmicos, classe antimicrobiana de amplo espectro, os quais foram prescritos em $18 \%$ dos pacientes (tendo 04 utilizado durante todo o internamento ertapenem e meropeneem - não simultâneos, 01 paciente apenas ertapeném e 70 somente meropenem). Devido ao alto perfil de multirresistência verificado durante a pesquisa, o quarto antimicrobiano mais prescrito foi a classe das polimixinas, sendo utilizado em $12,5 \%$ dos pacientes $(\mathrm{N}=52)$. Além do mais, $6 \%$ dos pacientes chegaram a fazer uso de ceftazidima-avibactam, um betalactâmico com potente ação sobre bactérias produtoras de carbapenemases do tipo serino-betalactamases $(\mathrm{N}=25)$. Destaca-se ainda, devido necessidade de associação com beta-lactamicos de amplo espectro para bactérias multirresistentes, o uso de aminoglicosídeos em cerca de 3,8 desses pacientes $(\mathrm{N}=16)$. Em relação às cefalosporinas, provavelmente devido ao tempo de internamento, analisamos apenas dois pacientes que fizeram uso de cefalosporina de terceira geração, três pacientes com o uso de cefalosporina de quarta geração e dez pacientes que foram prescritos com ceftarolina (Figura 5). 
Figura 5. Distribuição pelo número absoluto de antimicrobianos utilizados durante internação de pacientes infectados ou colonizados por bactérias multirresistentes em 2021.

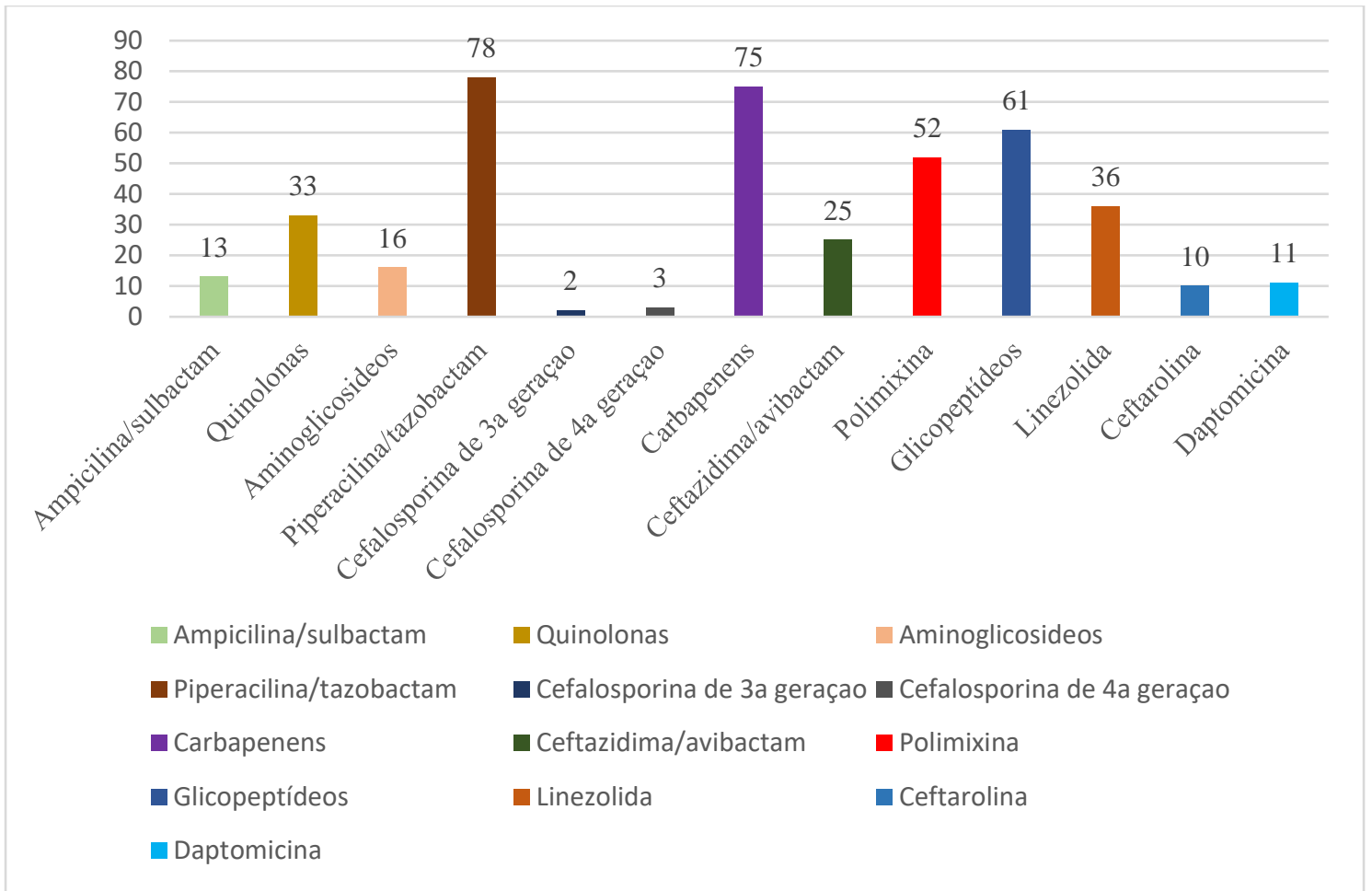

Fonte: Gráfico construído a partir de dados dos autores. Observa-se a prevalência da utilização elevada de betalactâmicos, carbapenêmicos e glicopeptídeos.

Quanto a associação entre as infecções relatadas e a mortalidade para estes pacientes, foi detectado que cerca de $44,8 \%$ dos pacientes foram a óbito durante o próprio internamento e 54,3\% foram de alta hospitalar ou transferidos. Dentre os três principais patógenos isolados e a elevada taxa de mortalidade dos mesmos, não houve diferença estatisticamente significativa em relação a taxa de mortalidade global para A. baumannii $(p=0,54), K$. pneumoniae $(p=1)$ e $P$. aeruginosa $(p=0,82),($ Figura $6)$.

Figura 6. Percentual de mortalidade durante internação de pacientes infectados ou colonizados por bactérias multirresistentes mais prevalentes durante o ano de 2021.

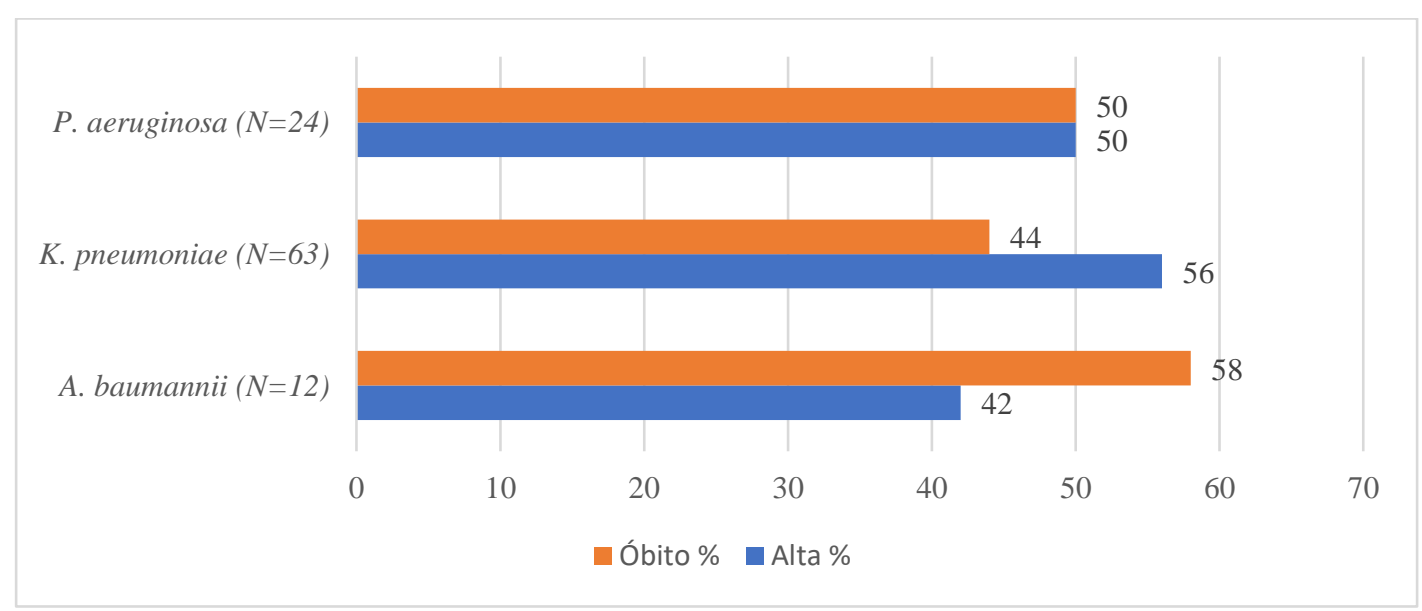

Fonte: Gráfico construído a partir de dados dos autores. Detecta-se uma maior mortalidade em percentual de pacientes infectados ou colonizados por A. baumannii, seguida por P. aeruginosa e K. pneumoniae. 
Ao avaliarmos o impacto do uso de antibióticos na mortalidade dos pacientes infectados ou colonizados por bactérias multirresistentes, detectou-se resultado estatisticamente significativo para utilização de quinolonas $(p=0,047)$ e uma tendência a significância para uso de polimixina ( $\mathrm{p}=0,09)$, sem resultados significativos para aminoglicosídeos, piperacilina/tazobactam, carbapenens, ceftazidima/avibactam ou glicopeptídeos (Figura 7).

Figura 7. Avaliação da mortalidade por utilização prévia de antimicrobianos durante internação no ano de 2021.

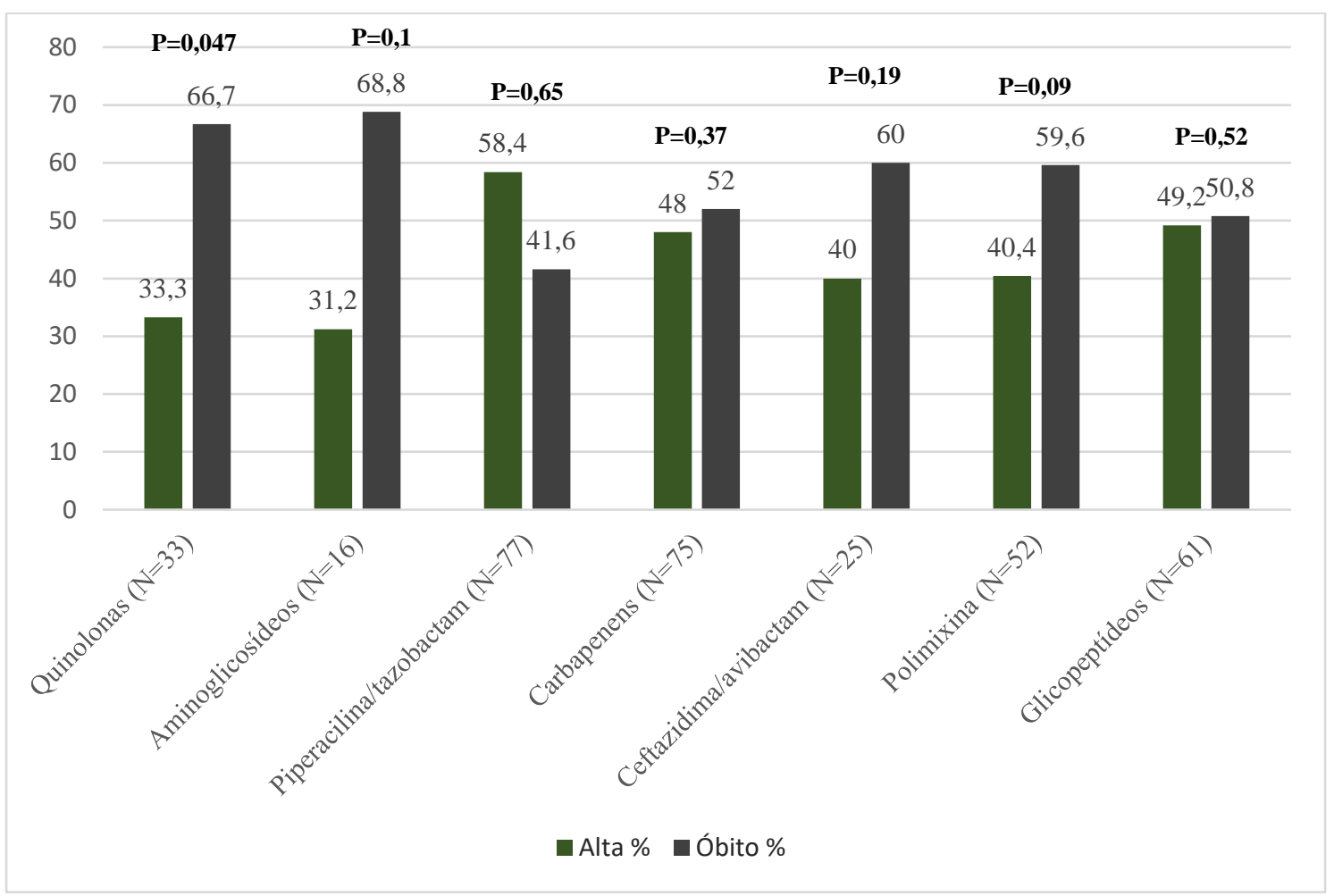

Fonte: Gráfico construído a partir de dados dos autores. Observa-se significância estatística para mortalidade associada a uso prévio de quinolonas dentre os antimicrobianos avaliados. Há um aumento de mortalidade com uso prévio de aminoglicosídeos, ceftazidima/avibactam e polimixinas, mas sem significância estatística.

\section{Discussão}

A faixa etária do presente estudo variou de 0 (dois pacientes recém-nascidos) a 103 anos, com média de 51,5 anos, sendo a faixa etária mais prevalente de 70 a 79 anos. E em comparação com estudo realizado anteriormente no Brasil, que evidenciou média de 59,7 anos, percebemos uma redução, inferindo que pacientes mais jovens podem estar apresentando maiores níveis de perfil para infecções por bactérias multirresistentes. Além disso, ressalta-se que vários pacientes foram internados por COVID-19 durante o ano de 2021, devendo ser considerado outras comorbidades que levaram estes pacientes ao internamento, como a obesidade, não mais presente em pacientes com idades mais avançadas, associadas a maior utilização de glicocorticoides impactando na sua resposta imune. Outras patologias crônicas mais conhecidas e evidenciadas mais fortemente na população de maior idade, como hipertensão arterial sistêmica e diabetes mellitus, podem justificar a diferença (Mota et al, 2018).

No resultado das amostras, o grande número de culturas positivas para microrganismos multirresistentes (74\%) foi através da coleta de swab retal, conforme protocolo interno de vigilância da instituição, definindo mais fatores de risco que a colonização por estes microrganismos trazem ao paciente do que propriamente as infecções per si, impactando em um menor número de pacientes com culturas positivas em outros sítios como hemoculturas, cultura do aspirado traqueal, cultura do lavado broncoalveolar e urocultura (26\% das amostras). Das principais bactérias que apresentam multirresistência K. pneumoniae é responsável por infecções hospitalares, comumente relacionadas a utilização de dispositivos invasivos em unidade de terapia 
intensiva, estando entre as principais bactérias isoladas nos exames. E. coli é normalmente encontrada como parte da microbiota normal do intestino grosso do homem e mantendo sua microbiota residente. Porém, algumas cepas dessa espécie são patogênicas. Streptococcus pneumoniae tem a recombinação genética como processo comum, onde um trecho do DNA exógeno é incorporado ao genoma, o que se representa vantajoso mecanismo de resistência. Pseudomonas aeruginosa é classificada como bacilo Gram negativo não fermentador de glicose, estando associada a infecções nosocomiais em pacientes imunodeprimidos e em ventilação mecânica. No Brasil, é comum causar infecções de sítios cirúrgicos, queimaduras e até septicemias graves. Sendo o gene envolvido na resistência aos antimicrobianos betalactâmicos o bla SPM-1, encontrado em isolados de UTIs (Santos et al., 2015; Machado et al., 2011).

Em estudo realizado em hospital do Nordeste, os microrganismos mais comumente isolados nas enfermarias foram os gram-negativos: Pseudomonas aeruginosa (61,5\%), seguido por Escherichia gergoviae (55,6\%) e Acinetobacter baumannii $(53,8 \%)$. Os micro-organismos mais isolados na UTI foram os Gram-negativos: Acinetobacter baumannii (46,2\%), seguidos da Escherichia gergoviae $(44,4 \%)$ e da Pseudomonas aeruginosa (38,5\%). Além disso, a proporção de pacientes que são resistentes a quatro ou mais drogas foi 1,26\% vezes maior na enfermaria do que na UTI (57,6 e 42,4 respectivamente), sendo as infecções respiratórias as mais prevalentes (Souza, 2020).

O principal sítio de infecção após o swab retal foi o pulmonar (aspirado traqueal e lavado broncoalveolar). Em um estudo sobre a prevalência e perfil de infecções em UTI no mundo, participando 1.150 centros em 88 países, em que o Brasil foi o terceiro país com maior inclusão de pacientes, observou-se que a infecção do trato respiratório teve maior prevalência com $60 \%$ dos casos (Vicent et al., 2020).

O principal patógeno em nosso estudo, envolvido em colonização e detecção via swab retal foi $K$. pneumoniae. Um estudo recente relata um índice de colonização de 6\% da amostra estudada, sendo que $67 \%$ desta teve como incidência a Klebsiella spp. Dos principais fatores que contribuíram para o desenvolvimento de colonização e de resistência bacteriana ressalta-se a prevalência de idosos $(74,28 \%)$, uso de dispositivos invasivos (100\%), tempo de uso de antibiótico maior que 7 dias $(42,4 \%)$ e tempo de internação maior que 48h (95,17\%), (Gabriel et al, 2021).

Detectou-se um impacto importante na mortalidade de pacientes com isolados de A. baumannii. A resistência do Acinetobacter spp. está diretamente relacionada ao impacto da utilização de múltiplos antibióticos direcionados para tratamento de infecções nosocomiais. Durante a última década, o tratamento dessas infecções tem se tornado crítico, em função do surgimento de cepas multirresistentes que podem ser disseminadas através da contaminação de equipamentos hospitalares (respiradores, ar-condicionado, equipamentos para diagnóstico por imagem, endoscopia e broncoscopia, dentre outros não adequadamente descontaminados) e/ou através da colonização da equipe assistencial (Gurgel, 2018). No estudo realizado no Nordeste, o Acinetobacter baumannii evidenciou perfil de resistência ao imipenem de 36,0\% e 47,1\%, nas enfermarias e UTI, respectivamente (Souza et al, 2020).

Um estudo com banco de dados que continha 355.116 pacientes, dos quais $30.561(8,6 \%)$ desenvolveram pelo menos um episódio de pneumonia adquirida na UTI, um total de 25.096 pacientes tinha infecção documentada e, para 18.529, um perfil bacteriano das cepas isoladas correspondentes a S. aureus, Enterobacteriaceae, P. aeruginosa ou A. baumannii estava disponível. Dos 18.497 casos de infecção incluídos, 3.081 (17\%) estavam infectados por bactérias multirresistentes. A mortalidade na UTI foi de 32\%, representando 5.872 pacientes de $68 \pm 13$ anos com Simplified Acute Physiology Score (SAPS 2) médio de $55 \pm 18$. O tempo médio de permanência na UTI foi de $33 \pm 26$ dias. Em subgrupos pré-especificados: mulheres versus homens, idade inferior a 65 anos versus superior (ou igual) a 65 anos, antibiótico na admissão na UTI versus sem antibiótico na admissão na UTI, paciente clínico versus paciente cirúrgico, ventilação mecânica versus sem ventilação mecânica e hospitalização paciente versus paciente fora do hospital, a infecção por bactéria multirresistente foi fator preditor de maior mortalidade (Lakbar et al, 2021). Estes dados corroboram o impacto desses fatores na mortalidade do presente estudo, porém a mortalidade neste último 
foi maior, principalmente em faixas etárias mais elevadas. Tal constatação pode ter sido relacionada ao impacto da pandemia por Covid-19, agravando o quadro pulmonar dos pacientes e pela exacerbação inflamatória propiciando um meio mais favorável a colonização e infecção bacteriana secundária.

Três estudos sugeriram que a resistência aos antibióticos levou a um aumento na mortalidade bruta, mesmo após o ajuste para dois deles. No primeiro estudo, a colonização por Enterobacter spp. ou espécies de K. pneumoniae foi fator preditivo independente para pneumonia em pacientes colonizados por bactérias produtoras de betalactamase de espectro estendido (ESBL), [OR 10,96 (2,93-41,0)], enquanto o recebimento de >2 dias de amoxicilina/ácido clavulânico durante a internação na UTI era fator protetor [OR 0,24 (0,08-0,71)]. Além de definir fatores como Insuficiência renal crônica, administração de cefalosporina de terceira geração nos últimos 3 meses, síndrome do desconforto respiratório agudo antes da pneumonia e terapia prévia com carbapenem ou fluoroquinolonas como associadas à pneumonia por Bactéria Resistente a Carbapenem (BRC) nesta população selecionada (Razazi et al, 2017). No segundo estudo, após avaliar 13.292 pacientes, evidenciaram que no subgrupo de Klebsiella spp. resistente a carbapenem houve mais óbitos do que o previsto (razão de mortalidade padronizada 1,20; IC 95\% 1,08-1,31), indicando um papel prognóstico negativo da infecção, principalmente nos pacientes de médio e alto risco de óbito. Quando a infecção foi causada por Klebsiella spp. ESBL, mas sensível a carbapenem, o tratamento com piperacilina-tazobactam aumentou a mortalidade ajustada entre os pacientes mais graves (semelhante ao grupo resistente a carbapenem), enquanto o tratamento com carbapenêmicos não (Bertolini et al, 2018). Portanto, a escolha correta de antimicrobiano na terapia empírica foi o diferencial quanto ao desfecho negativo nos pacientes com alto risco de colonização ou infecção por bactérias multirresistentes. No terceiro estudo, entre 16.734 pacientes avaliados, a exposição ao carbapenem aumentou em portadores infectados e não infectados por bactérias multirresistentes quando comparados com não portadores (627, 241 e 69 dias de carbapenem por 1.000 pacientes-dia, respectivamente, $\mathrm{P}<0,001)$, com colonizados por Enterobacteriacea produtora de ESBL, prolongamento do tempo de internação e favorecimento de pressão seletiva responsável por aumento de resistência nas UTIs (Barbier et al, 2016). Portanto, com o aumento no nosso estudo de uso de carbapenens, justifica-se o impacto na alta mortalidade encontrada.

Alguns mecanismos de resistência como transmissão plasmidial podem ser responsáveis por grandes surtos e mortalidades em hospitais. Como o blaKPC-2 que foi a única carbapenemase detectada em surto de hospital universitário na China. Os principais genes de virulência foram uge (100\%), $\operatorname{mrkD}(94,1 \%)$, kpn $(94,1 \%)$ e fim-H $(72,5 \%)$, enquanto os genes wcag, ironB, alls e magA não foram detectados. Uma cepa do tipo sequência ST1373, K. pneumoniae hipervirulenta (hvKP), foi detectada. As cepas de Klebsiella pneumoniae produtora de carbapenemase (CRKP) foram altamente resistentes a quinolonas, cefalosporinas, aminoglicosídeos e polimixina, mas suscetíveis a tigeciclina e ceftazidima-avibactam. Em protocolos e instituições onde a polimixina seria a última estratégia terapêutica teriam desfechos negativos diante de uma disseminação de virulência semelhante (Zeng et al, 2021). Portanto, é de extrema importância que os laboratórios comecem a trabalhar não apenas com o mecanismo de resistência, mas também com a identificação das alterações enzimáticas por sequenciamento genético.

A despeito da variabilidade brasileira em relação a etnia, condição socioeconômica, hábitos e estilo de vida, e levando em consideração o papel dos centros de controle de infecção hospitalar em cada unidade, os estudos de prevalências em grande escala são necessários para detectar os principais agentes patogênicos nos quais as pesquisas futuras precisam dar enfoque e as estratégias de saúde pública devem se voltar (de Carvalho et al., 2021).

Existe ainda muita fragilidade e inconsistências na execução dos protocolos de segurança e higiene, realizados por equipes responsáveis pelo cuidado da saúde da população, intra e extra-hospitalares. O que pode ser visto diretamente na redução do combate às doenças bacterianas, impacto econômico elevado, incorrendo em grandes gastos hospitalares e aumento da morbidade e mortalidade dos indivíduos (de Carvalho et al., 2021). 
Com o aumento crescente das bactérias multirresistente, inversamente proporcional a diminuição do número de antibióticos para combatê-las, impulsionam a necessidade de estratégias voltadas para redução dessas infecções (da Silva, 2020). Uma importante estratégia é controlar o tempo de exposição aos antibióticos na UTI, minimizando o uso desnecessário desses agentes. Tais abordagens estarão cada vez mais ligadas a avanços em testes de microbiologia molecular e inteligência artificial/aprendizado de máquina. Esses avanços devem ajudar a identificar os pacientes que necessitam de antibioticoterapia empírica em um momento anterior, bem como os antibióticos específicos necessários para evitar a administração desnecessária de antibióticos de amplo espectro (Kollef et al, 2021).

\section{Considerações Finais}

O estudo evidenciou elevada mortalidade em pacientes colonizados ou infectados por bactérias multirresistentes em âmbito hospitalar, principalmente em faixas etárias acima de 70 anos de idade, o que pode ser responsável por menor tempo de permanência hospitalar nessa subpopulação.

As bactérias Gram negativas foram as mais prevalentes, principalmente K. pneumoniae, P. aeruginosa e A. baumannii, com tendência a maior mortalidade pela última. $\mathrm{O}$ uso de quinolonas, aminoglicosídeos e polimixina parece estar relacionado a maior mortalidade.

Além disso, mais e maiores estudos sobre o perfil de suscetibilidade e prevalência microbiana são necessários para traçar estratégias de saúde pública voltadas ao controle da farmacorresistência. Assim, são necessárias análises posteriores, e em larga escala, de estratégias educativas populacionais e das equipes multidisciplinares de linha de frente, bem como planejamentos governamentais voltados à saúde pública, evitando os riscos de negligenciar essa situação delicada e perigosa: a multirresistência bacteriana.

Algumas recomendações para reduzir a incidência das infecções relacionadas aos serviços de saúde seriam: vigilância epidemiológica diária do uso dos antibióticos, educação permanente com as equipes multidisciplinares com protocolos rígidos de antibioticoterapia preconizado pela instituição, implantação de barreira na prescrição médica depois do vencimento das doses adequadas para o tratamento, educação permanente com as equipes multidisciplinares para que se tenha uma maior cooperação com o Serviço de Controle de Infecção Hospitalar tanto para que o número de subnotificações dos casos diminua, quanto para uma melhora da qualidade do cuidado.

\section{Agradecimentos}

Agradecemos a todo o Serviço de Controle de Infecção Hospitalar (SCIH) do Hospital São Camilo de Fortaleza, principalmente Matheus César Dias Patrício, Francelene da Silva Almeida, Patrícia Borges Barjud Coelho e Virna Yane Bezerra Lopes, com os quais seria impossível enfrentar uma pandemia de tamanha magnitude. Time cuja dedicação e competência não apresentam precedentes.

\section{Referências}

ANVISA, Nota Técnica GVIMS/GGTES/ANVISA No 05/2021; Orientações para prevenção e controle da disseminação de microrganismos multirresistentes em serviços de saúde no contexto da pandemia da COVID-19 - 02/08/2021.

Barbier, F., Pommier, C., Essaied, W., Garrouste-Orgeas, M., Schwebel, C., Ruckly, S., Dumenil, A. S., Lemiale, V., Mourvillier, B., Clec'h, C., Darmon, M., Laurent, V., Marcotte, G., Lucet, J. C., Souweine, B., Zahar, J. R., Timsit, J. F., \& OUTCOMEREA Study Group (2016). Colonization and infection with extended-spectrum $\beta$-lactamase-producing Enterobacteriaceae in ICU patients: what impact on outcomes and carbapenem exposure? The Journal of antimicrobial chemotherapy, 71(4), 1088-1097. https://doi.org/10.1093/jac/dkv423

Bassetti, M., Merelli, M., Temperoni, C., \& Astilean, A. (2013). New antibiotics for bad bugs: where are we? Annals of clinical microbiology and antimicrobials, 12, 22. https://doi.org/10.1186/1476-0711-12-22 
Bassetti, M., \& Righi, E. (2015). New antibiotics and antimicrobial combination therapy for the treatment of gram-negative bacterial infections. Current opinion in critical care, 21(5), 402-411. https://doi.org/10.1097/MCC.0000000000000235

Bassetti, M., \& Righi, E. (2015). Development of novel antibacterial drugs to combat multiple resistant organisms. Langenbeck's archives of surgery, 400(2), 153-165. https://doi.org/10.1007/s00423-015-1280-4

Bertolini, G., Nattino, G., Tascini, C., Poole, D., Viaggi, B., Carrara, G., Rossi, C., Crespi, D., Mondini, M., Langer, M., Rossolini, G. M., \& Malacarne, P. GiViTI Steering Committee. (2018). Mortality attributable to different Klebsiella susceptibility patterns and to the coverage of empirical antibiotic therapy: a cohort study on patients admitted to the ICU with infection. Intensive care medicine, 44(10), 1709-1719. https://doi.org/10.1007/s00134-018-5360-0

Boucher, H. W., Talbot, G. H., Bradley, J. S., Edwards, J. E., Gilbert, D., Rice, L. B., Scheld, M., Spellberg, B., \& Bartlett, J. (2009). Bad bugs, no drugs: no ESKAPE! An update from the Infectious Diseases Society of America. Clinical infectious Diseases: an official publication of the Infectious Diseases Society of America, 48(1), 1-12. https://doi.org/10.1086/595011

Costa, A. L. P. (2016) Resistência Bacteriana aos Antibióticos: Uma Perspectiva Do Fenômeno Biológico, Suas Consequencias e Estratégias De Contenção. 63 f. Trabalho de Conclusão de Curso (Graduação em Biologia) - Curso de Ciências Biológicas, Departamento de Ciências Biológicas e da Saúde, UNIFAP, Macapá.

Da Silva, H. R., Rocha, A. S. C., Rocha, M. V. S., Veras, D. M., de Sousa, O. M. C., Sousa, G. C., Almeida, D. F., de Oliveira, A. L. P., Bezerra, G. S. S., Ribeiro, D. A. S., Pereira, N. M., Alves, A. K. R., Alves, A. K. R., da Silva, B. B. L., Nogueira, F. D., Rodrigues, S. L. D., \& Pessoa, G. T. (2020). Reflection of environmental imbalance in health: multi-resistant bacteria in hospital environment. Research, Society and Development, 9(8), e220985604.

De Carvalho, J. J. V., Boaventura, F. G., da Silva, A. C. R., Ximenes, R. L., Rodrigues, L. K. C., Nunes, D. A. A., \& de Souza, V. K. G. (2021). Bactérias multirresistentes e seus impactos na saúde pública: Uma responsabilidade social. Research, Society and Development, 10(6), e58810616303

De Queiroz, G. M., da Silva, L. M., Pietro, R. C. L. R., \& Salgado, H. R. N. (2012). Multirresistência microbiana e opções terapêuticas disponíveis / Microbial multi-resistance and available therapeutic options. Rev Bras Clin Med. 10(2), 132-8.

Gabriel, A. C. G., Pinheirp, D. H. P., Fernnades, L. M. S., Wind, M. M., Ferreira, V. R., \& Pereira, M. S. (2021). Colonization profile and microbiological resistance in Intensive Care Unit (ICU) patients in the city of Anápolis - GO. Research, Society and Development, 10(16), e588101623853

Gurgel, T. C., \& Carvalho, W. S. (2018). A Assistência Farmacêutica e o Aumento da Resistência Bacteriana aos Antibióticos. Latin American Journal of Pharmacy, 27(1), 118-123

Kollef, M. H., Shorr, A. F., Bassetti, M., Timsit, J. F., Micek, S. T., Michelson, A. P., \& Garnacho-Montero, J. (2021). Timing of antibiotic therapy in the ICU. Critical care (London, England), 25(1), 360. https://doi.org/10.1186/s13054-021-03787-z

Langford, B. J., So M., Raybardhan S., Leung V., Soucy J. R., Westwood D., Daneman N., MacFadden, D. R. (2021). Antibiotic prescribing in patients with COVID- 19: rapid review and meta-analysis. Clinical Microbiology and Infection, 27, 520e531.

Lakbar, I., Medam, S., Ronflé1, R., Cassir, N., Delamarre, L., Hammad, E., Lopez, A., Lepape, A., Machut, A., Boucekine, M., Zieleskiewicz, L., Baumstarck, K., Savey, A., Leone, M. \& REA RAISIN Study Group. (2021). Association between mortality and highly antimicrobial-resistant bacteria in intensive care unitacquired pneumonia. Nature Scientific Reports, 11:16497 https://www.nature.com/srep/

Machado, G. M., Lago, A., Fuentefria, S. R. R. \& Fuentefria, D. B. (2011). Occurrence and the susceptibility to antimicrobial agents in Pseudomonas aeruginosa and Acinetobacter sp. at a tertiary hospital in southern Brazil. Rev. Soc. Bras. Med. Trop. 44(2), 168-172.

Mota, F. S., Oliveira, H. A., \& Souto, R. C. F. (2018). Perfil e prevalência de resistência aos antimicrobianos de bactérias Gram-negativas isoladas de pacientes de uma unidade de terapia intensiva. RBAC. 50(3):270-7. DOI: 10.21877/2448-3877.201800740

Nascimento, C. K. R., Santos, M. M., Oliveira, P. M. C., Iomori, M. V. A. H., Santos, R. M. M., Martins, V. B. D., Melo, B. F., Nascimento, A. L. L., Santos, I. S. P., Dias, T. C., Santos, M. H. S., Rodrigues, A. A., Bezerra, M. S. L., Andrade, R. S. B., \& Santos, S. C. (2021). Microbiological and immunological aspects related to the colonization and infection of patients by resistant Acinetobacter baumannii in nosocomial environment. Research, Society and Development, $\mathrm{v}$. 10, n. 2, e25910212650

Queenan, K., Hasler, B. J. \& Rushton, A. (2014). One Health approach to antimicrobial resistance surveillance: is there a business case for it? International Journal of Antimicrobial Agents, Volume 48(4).

Ranganathan, P., \& Aggarwal, R. (2018). Study designs: Part 1 - An overview and classification. Perspectives in clinical research, 9(4), 184-186. https://doi.org/10.4103/picr.PICR_124_18

Razazi, K., Mekontso Dessap, A., Carteaux, G., Jansen, C., Decousser, J. W., de Prost, N., \& Brun-Buisson, C. (2017). Frequency, associated factors and outcome of multi-drug-resistant intensive care unit-acquired pneumonia among patients colonized with extended-spectrum $\beta$-lactamase-producing Enterobacteriaceae. Annals of intensive care, 7(1), 61. https://doi.org/10.1186/s13613-017-0283-4

Santos, I. A. L., Nogueira, J. M. R. \& Mendonça, F. C. R. (2015). Mecanismos de resistência antimicrobiana em Pseudomonas aeruginosa. RBAC. 47(1-2), 512.

Souza, E. E. S., Almeida, A. G. C. S., de Souza, C. D. F., Nagliate, P. C., Borges, K. R., \& Junges, A. V. (2021). Microbial resistance profile of infections related to health care in a hospital for infectious diseases in a capital of Northeastern Brazil. Research, Society and Development, 10(4), e39910414198.

Vincent, J. L., Sakr, Y., Singer, M., Loeches, I. M., Machado, F. R., Marshall, J. C, Finfer, S. Pelosi, P., Brazzi, L., Aditi aningsih, D., Timsit, J. F., Du, B., Wittebole, X., Máca, J., Kannan, S., Delsol, L. A. G., Waele, J. J. D., Mehta, Y., Bonten, M. J. M., Khanna, A. K., Kollef, M., Human, M., \& Angus, D. C. (2020). Prevalence and Outcomes of Infection Among Patients in Intensive Care Units in 2017. JAMA. 323(15):1478-1487. 10.1001/jama.2020.2717. https://jamanetwork.com/journals/jama/fullarticle/2763669

WHO, New report calls for urgent action to avert antimicrobial resistance crisis. 2019. https://www.who.int/news/item/29-04-2019-new-report-calls-for-urgentaction-to-avert-antimicrobial-resistance-crisis

Zeng, L., Yang, C., Zhang, J., Hu, K., Zou, J., Li, J., Wang, J., Huang, W., Yin, L. \& Zhang, X. (2021). An Outbreak of Carbapenem- Resistant Klebsiella pneumoniae in an Intensive Care Unit of a Major Teaching Hospital in Chongqing, China. Frontiers in Cellular and Infection Microbiology, June, Volume 11, Article 656070. 